\title{
Location Prediction Weighted Clustering Algorithm Based on WNNM in Ad Hoc Network
}

\author{
Sha Yi,Huang Li
Northeastern University \\ Sha Yi,Huang Li
Northeastern University
}

\begin{abstract}
Introducing a wavelet neural network model (WNNM) at a route maintenance stage to predict the position of nodes in ad hoc networks, a new weighted clustering algorithm (WCA) is presented. Using the nodes' position information from GPS, this algorithm predicts the position of nodes at next time by WNNM, then calculates the time the neighbor nodes spend moving out of the coverage of cluster heads by the predicted values and takes it as a measurement of aggregate holding time. If the cluster structure tends to be unstable, a pre-repair mechanism will function before the link fails, thus avoiding frequent break of links and improving the network performance. Simulation results show that compared to the Lowest-ID WCA and Location-based WCA, the algorithm proposed can increase the packet delivery rate by $9 \%$ and $6 \%$, respectively, and decrease the numbers of link break by about $70 \%$ and $50 \%$, respectively.
\end{abstract}

Keywords-Ad hoc network, Weighted clustering algorithm (WCA), Location prediction, Wavelet neural network model (WNNM)

\section{INTRODUCTION}

Ad hoc is a special mobile network which combining mobile communication and computer network [1]. Each node of the network not only has functions as a common terminal, but also forwards packet. At first, the structure of an ad hoc network is two dimensional, and every node holds the route information with the other nodes. With the sending data increases, the node has to bear more and more overload. One of the best methods to solve this problem is to use the cluster structure [2]. Every cluster is composed of one head and some common nodes. Then, the head and the gateway form the high priority virtual backbone network (VBN). The function of cluster members is simple. In contrast, the head takes in charge of intra-cluster or inter-cluster communication. Therefore, how to select a head node will affect the stability and reliability of networks.

Now, lots of research has been done on the cluster assignment algorithm (CA) of ad hoc networks. In [3], a weighted clustering algorithm (WAC) has been proposed, which allocates the weight allocated, according to the battery energy, node speed, cluster coverage and numbers of neighbors. Then, calculate the combination weights, and chose the lowest one as the head. In [4], WCA has been improved. Prediction is not involved in the above algorithms, which can fit the dynamic topology of ad hoc network. As the network topology changes fast, the cluster structure will be unstable. In [5], a cluster algorithm with location prediction has been proposed. However, it doesn't insert prediction model, as a result, it can not acquire the link's

\author{
Chu Jiafu,Zhang Lili \\ Northeastern University
}

future state.

Aiming at the above problems, this paper presents a cluster algorithm which is based on the prediction of node's aggregate hold time. During the route maintenance phase, the algorithm predicts the node's position by WNNM, with the help of the GPS location information. And then, we can obtain the aggregate hold time which can be used to judge whether the link is stable. Before the link fails, a pre-repair process will start to avoid frequent link failures. Thus, the influence of dynamic topology can be reduced in order to improve the whole performance of the whole network.

\section{WAVELET NeURAL NETWORK MOdEL(WNNM)}

\section{A. introduction of WNNM}

The mobility of the node is related with the specific application and, as a result, it always has regularity and purposiveness. Therefore, it is possible to predict the motion path of the node. Because of the complex non-linear of most system, traditional prediction method has certain limitation, both theoretically and practically. In [6], the prediction based on WNNM has been proved as an effective method to solve non-linear issues.

The WNNM is a kind of local basis function network model, whose structure and expression are almost the same as the BP neural network. The difference between the two models mainly lies in that the wavelet function $\psi(t)$ is chosen as the hidden layer excitation function to substitute the BP excitation sigmoid function. $\psi(t)$ changes with the practical need. The Sigmoid function is chosen as output excitation function, whose expression is $f(x)=1 /[1+\exp (-x)]$

The wavelet function in this paper is the Morlet, widely used both home and abroad. The expression is as follow:

$\phi(x)=\cos (1.75 x) e^{-x^{2} / 2}$

The output of WNNM is as follows:

$$
y_{i}=f\left(\sum_{j=1}^{m} \omega_{i j} \psi\left(\frac{\sum_{k=1}^{n} \omega_{j k} x_{k}-b_{j}}{a_{j}}\right)\right)
$$

In which, $\mathrm{x}$ represents $\mathrm{n}$ dimensional enter vector, $x=\left\{x_{k} \mid x_{k} \in X, k=1,2, \ldots, n\right\}, \mathrm{X}$ represents sequence of inputs, $\mathrm{n}$ represents the input number of wavelet operators. The weight between input and hidden layer is $\boldsymbol{\omega}_{i j}$, and that 
between output and hidden layer is $\boldsymbol{\omega}_{j k}$. The stretching and translation parameter of middle hidden layer are $a_{j}$ and $b_{j}$, respectively.

\section{B. sample extraction and netword training}

In Ad Hoc network, the position of the node varies as the time elapses. This paper gets the location between the same interval in NS2 to build up the sequence of samples. In order to eliminate the dimension and fluctuation of the data, the sample needs pretreatment and aftertreatment:

$$
X=\left\{x_{j}\right\}, x_{j}=\frac{s_{j}-s_{\min }}{s_{\max }-s_{\min }}, s_{j} \in S, j=1,2, \ldots, N
$$

In which, $S_{\max }$ represents the $\max$ sample, $S_{\text {min }}$ represents the minimal sample. Then, normalized samples need dividing into groups. $\mathrm{X}$ will be converted into a matrix with $n$ rows and $\mathrm{N}-\mathrm{n}+2$ columns, which is as follows:

$$
X=\left[\begin{array}{cccc}
x_{1} & x_{2} & \cdots & x_{n} \\
x_{2} & x_{3} & & x_{n+1} \\
\vdots & & \ddots & \vdots \\
x_{N-(n-2)} & x_{N-(n-3)} & \cdots & x_{N-1}
\end{array}\right]
$$

In which, $\mathrm{N}$ represents sample numbers, $\mathrm{n}$ represents numbers of wavelet neurons. Thus, we can acquire the $\mathrm{N}-\mathrm{n}+2$ couples of sample inputs and outputs, and then proceed with the network training.

After confirming the structure of WNNM, only the weight can be changed. Because the outputs of wavelet network and the weight are linearity, the orthogonal least square (OLS) method, with better robustness, should be adopted. The process of this algorithm is as follows:

For a wavelet neural, the contribution for network outputs can not acquire directly. However, after orthogonalization, the contribution can be got directly. First, assume the number of hidden layer nodes is equal to the input samples, and then take the error rate of decline as basis to pick the wavelet neural. At the same time, the weight between hidden layer and output layer should be calculated. In this way, the network not only is unique, but also holds minimal wavelet neural.

\section{WCA BASED ON LOCATION}

\section{A. Design method}

The position-information-based WCA (p-WCA), proposed by this paper, synthetically weighs the location information of the node, the distance between node and virtual cluster center $\left(V_{C C}\right)$ and the node degree to elect the reasonable cluster head. Therefore, the node combination weights can be calculated as follows:

$$
\mathrm{W}=-\omega_{1} \times \mathrm{T}+\omega_{2} \times D+\omega_{3} \times d_{v}
$$

(1) $\mathrm{T}$ : node aggregate holding time.

$$
T=\sum_{j=1}^{N} t_{j} t_{j}=\frac{\sqrt{\left(y_{1}-y_{j}\right)^{2}+\left(x_{1}-x_{j}\right)^{2}}}{|v|}
$$

The current position of node $\mathrm{i}$ is $\left(x_{i}, y_{i}\right)$, the speed is $\left(v_{x 0}, v_{y 0}\right)$; the position of neighbor node $\mathrm{j}$ relative to node $\mathrm{i}$ is $\left(x_{j}, y_{j}\right)$, so, node $\mathrm{j}$ 's speed relative to $\mathrm{i}$ is:

$$
\left(v_{x}, v_{y}\right)=\left(v_{x j}-v_{x 0}, v_{y j}-v_{y 0}\right)
$$

When $|v|$ is small but it isn't 0 , the relative hold time will be tremendously large, which will make the time factor too influential, and then weaken the other factor. So, a lowest speed threshold should be set as $v_{\min }$. When $|v|<v_{\text {min }}$, in expression (6), use $v_{\text {min }}$ to replace $|v|$. When $|v|_{=0, \text { make }} t_{j}=2 R / v_{\text {min }}$, which is exactly the max aggregate holding time under the node coverage. At last, the aggregate holding time $\mathrm{T}$, at which each neighbor node will move out of current node's communication coverage, will be calculated by the collective data. The larger of the $T$, the more possible the current node may be the cluster head.

(2)D: distance between node and $V_{C C}$.

$$
\begin{gathered}
D=\left|(x, y)-\left(x_{V_{C C}}-y_{V_{C C}}\right)\right| \\
\text { (3) } d_{v} \text { : node degree. } \\
d_{v}=|N(v)|=\sum_{v, v^{\prime} \in V, v \neq v^{\prime}}\left\{\text { dist }\left(v, v^{\prime}\right)<\text { range }\right\}
\end{gathered}
$$

In which, $v$ and $v^{\prime}$ are the node out of cluster. For node $v$ whose degree isn't zero, the offset between ${ }^{v}$ 's degree and the best node's degree can be calculated as follows:

$$
\Delta_{v}=\left|d_{v}-\delta\right|
$$

$\omega_{1}, \omega_{2}$ and $\omega_{3}$ are weight factor, satisfying $\omega_{1}+\omega_{2}+\omega_{3}=1$. In this algorithm, they are 0.6 , 0.2 and 0.2 respectively.

\section{B. procedure to build cluster}

The specific procedure is as follows:

- While initialization, each node will get one unique ID, allotted by system.

- According to the transmission radius and area size, virtual cluster will be divided. Every node gets the location information by GPS. Then, calculate the distance between $C_{C C}$ to make pre-clustering.

- Each node gets the neighbor's ID, the location and movement information by Hello packet and calculates the weight $\mathrm{W}$ afterwards. The message including W and node's ID will be broadcast to neighbors. After receiving the weight message, the 
node will change the neighbor table. Then, compare the neighbor's weight with that of the node itself. If even, compare the ID. If ID of itself is small, the node declare itself as the head, the neighbor as the cluster member. It goes on until all the nodes are assigned.

\section{P-WCA INSERTED WNNM}

\section{B. $\quad$ prediction of aggregate holding time}

Assume cluster head i, cluster member $\mathrm{j}$, the location information of previous $n-1$ epoches and current epoch $t$. The parameter of WNNM can be calculated by OLS method, then the node $i$ and $j$ 's location information at $t+1$ epoch can

be calculated, which is $\left(x_{i}(t+1), y_{i}(t+1)\right)$ and $\left(\hat{x}_{j}(t+1), \hat{y}_{j}(t+1)\right)$. Thus, $\hat{t}_{j}$ which is the time when node $\mathrm{j}$ will move out of $\mathrm{CH}$ node i's communication coverage can be expressed as follows:

$$
\hat{t}_{j}=\frac{\sqrt{\left(\hat{y}_{1}(t+1)-\hat{y}_{j}(t+1)\right)^{2}+\left(\hat{x}_{1}(t+1)-\hat{x}_{j}(t+1)\right)^{2}}}{|v|}
$$

$\left(\hat{x}_{1}(t+1), \hat{y}_{1}(t+1)\right)$ is the prediction value of intersection point $\mathrm{C}_{j} . \hat{T}=\sum_{j} \hat{t}_{j}$ is the prediction value of node aggregate holding time. The intersection point $\mathrm{C}_{j}$, where neighbor node $j$ moves out of the current cluster head's communication coverage, can be calculated by expression (7). Node aggregate holding time is ${ }^{T=\sum_{j} t_{j}} . \hat{T}<T$ represents that node $\mathrm{j}$ will move out the communication immediately, which means that the structure of the cluster will be unstable.

Now, $\mathrm{T}$ is the aggregate holding time threshold $T^{\prime}$.

\section{Route pre-recovery process and route mechanism}

AODV has been implemented in NS2. The WNNM-P-WCA adds parameter prediction function on the basis of P-WCA. In order to implement route prediction, a pre-recovery state should be involved. During the route maintenance, as soon as the cluster structure instability is predicted, the pre-recovery will be initiated. Thus, route can be chosen before failure.

As long as the node becomes a cluster member, the route discovery mechanism is as follows:

- If the source node who sends out RREQ is cluster member, it initializes RREQ packet, records the location information of itself and then check the route table to make sure whether the route to the current exists. If there is, use this route to unicast RREQ to cluster head, if not, broadcast RREQ.

- After the cluster receives RREQ, it checks whether this packet has been received. If not, checks whether the destination node is in the cluster. If there is , unicast to the destination node, if not, looks for the route to the destination node. If find it one, send
RREP to source node, if doesn't, broadcast to neighbor cluster head.

- The nodes who receive RREP, first check whether this packet has been received before. If the node isn't cluster head, forward the RREP, if it is, finds the route to source node. If fails, establish a reverse one, if successes, update the route table. At last, if the source node is in the current cluster, unicast the RREP, if not, forward RREP to neighbor cluster head.

The cluster AODV protocol, on the one hand, periodically broadcasts HELLO message to assert neighbor, on the other hand, take the aggregate holding time ${ }^{\mathrm{T}_{0}{ }^{\prime}}$ as a warning symbol. If the prediction value of aggregate holding

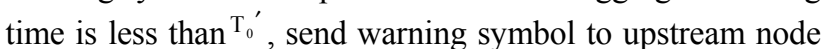
and initialize route maintenance process. After previous node has established a new route, switch the link which is more stable with data transmission unbroken.

\section{Simulation Results}

This paper uses NS2 to implement the WNNM-P-WCA, P-WCA and Lowest-ID WCA. Table 1 shows the environment parameters during the simulation. The initialized location is random. The topology is changing every time because of no stop time. Each node uses the same wireless equipment, whose communication radius is $250 \mathrm{~m}$ and channel capacity is $2 \mathrm{Mbit} / \mathrm{s}$.

In order to comprehensively analyze the performance of WNNM-P-WCA, P-WCA and Lowest-ID WCA, this paper uses the following measurements:

- Packet delivery rate.

- The number of link interruption.

- Normalized routing overhead.

With the speed of the node changing from $1 \mathrm{~m} / \mathrm{s}$ to $20 \mathrm{~m} / \mathrm{s}$, the packet delivery rates of these algorithms are above $85 \%$, although they are all decreasing, as showed in Figure 1. WNNM-P-WCA is $9 \%$ higher than Lowest-ID WCA, $6 \%$ higher than P-WCA. This is because that it adds pre-recovery process during route maintenance before route interruption.

The normalized routing overhead is proportional to the speed. When the speed is fixed, the overhead of WNNM-P-WCA is slightly large, as showed in Figure.2. This is because that during the route maintenance, WNNM-P-WCA adds warning packet. However, relative to the reduction of packet delivery rate and number of link interruption, it is acceptable. When the speed increases, the number of link interruption increases. But WNNP-P-WCA is obviously small than P-WCA and Lowest-ID WCA. It is $70 \%$ less than Lowest-ID WCA and 50\% less than P-WCA, as showed in Figure. 3.

\section{SUMMARIES}

This paper presents WNNM-P-WCA, who uses WNNM to predict the location information of nodes. According to the result, cluster head aggregate holding time can be calculated. In this way, the pre-recovery can be initialized before the link fails. Simulation results show that 
number of link interruption of WNNM-P-WCA is 221 less than P-WCA, 419 less than Lowest-ID WCA. The packet delivery rate is $9 \%$ higher than $\mathrm{P}-\mathrm{WCA}$ and $6 \%$ higher than Lowest-ID WCA. Meanwhile, the performance advantage of WNNM-P-WCA is more obvious as the speed increases.

\section{REFERENCES}

[1] Frodigh M, Johansson P, Larsson P. Wireless ad hoc networking - the art of networking without a network[J]. Ericsson Review, 2000 (4):248-262.

[2] Remondo D, Niemegeers I G. Ad hoc networking in future wireless communications [J]. Computer Communications, 2003, 26(1): 36-40.
[3] Basu P, Khan N, Little T D C. A mobility based metric for clustering in mobile ad hoc networks[C]// Proceedings of IEEE ICDCS 2001 Workshop on Wireless Networks and Mobile Computing. Phoenix: IEEE, 2001:413-418.

[4] Chatterjee M, Das S K, Turgut D. WCA: A weighted clustering algorithm for mobile ad hoc networks[J]. Cluster Computing, 2002, 5(2):193-204

[5] Chatterjee M, Das S K, Turgut D. An on-demand weighted clustering algorithm (WCA) for ad hoc networks[C]// Proceedings of GLOBECOM 2000, San Francisco: IEEE, 2000: 1697-1701.

[6] Rezaee M, Yaghmaee M H. A new clustering protocol for mobile ad hoc networks[C]//nternational Symposium on Telecommunications, 2008: 376-381.

TABLEI. PARAMETERS OF SIMULATION

\begin{tabular}{|c|c|c|c|}
\hline Parameter & Value & parameter & value \\
\hline Route protocol & AODV & Propagation type & TwoRayGround \\
\hline Channel & Wireless Channel & Interface & Phy/WirelessPhy \\
\hline Motion type & RWP & Service type & CBR \\
\hline Speed & $1,5,10,15,20 \mathrm{~m} / \mathrm{s}$ & Node number & 30 \\
\hline Scene & $800 \mathrm{~m} * 800 \mathrm{~m}$ & Connection number & 10 \\
\hline Simulation time & $600 \mathrm{~s}$ & Packet rate & $1 \mathrm{packet} / \mathrm{s}$ \\
\hline Queue & PriQueue & Queue length & 50 \\
\hline
\end{tabular}
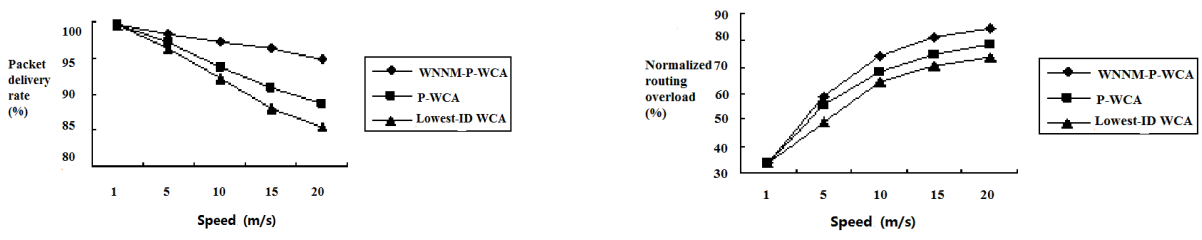

Figure1. Packet delivery rate

Figure2. Normalized routing overload

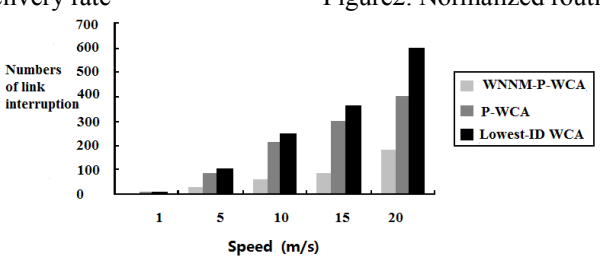

Figure3. Numbers of link interruption 